\title{
Lymphatic Microvessel Density and the Expression of Lymphangiogenic Factors in Oral Squamous Cell Carcinoma
}

\author{
Mohammad A. Ali \\ Department of Diagnostic Sciences, Faculty of Dentistry, Kuwait University, Kuwait
}

\section{Key Words}

Oral squamous cell carcinoma $\cdot$ Lymphangiogenesis .

Lymphangiogenic growth factors - Lymphatic microvessel density

\begin{abstract}
Objective: In this study, the expression of several lymphangiogenic growth factors was investigated to determine the mechanism underlying lymphangiogenesis in oral squamous cell carcinoma (OSCCa). Materials and Methods: An immunohistochemical study was performed to determine the lymphatic microvessel density (LMVD) and the expression of vascular endothelial growth factor (VEGF), plateletderived growth factor (PDGF), basic fibroblast growth factor (bFGF), insulin-like growth factors (IGF-I/II), and hepatocyte growth factor (HGF) in 25 OSCCa and 20 normal oral mucosal tissue samples. Results: Significantly higher levels of expression of VEGF, PDGF, bFGF, HGF, IGF-I, and IGF-II existed in OSCCa tissue compared to normal oral mucosal tissue $(p<$ $0.001, p<0.001, p<0.001, p<0.001, p<0.001$, and $p<0.01$, respectively; Mann-Whitney test). In OSCCa samples, significant positive correlations, based on the Spearman correlation test, existed between the LMVD and VEGF $(r=0.573$; $p<0.01)$, PDGF $(r=0.559 ; p<0.01)$, bFGF $(r=0.516 ; p<0.01)$, HGF $(r=0.448 ; p<0.05)$, and IGF-I $(r=0.6 ; p<0.01)$, but no correlation existed between LMVD and IGF-II $(r=0.380$; $p>0.05)$. Conclusion: These findings indicate that several
\end{abstract}

growth factors may promote lymphangiogenesis in OSCCa. Future treatment for OSCCa utilizing antilymphangiogenic agents should target these different growth factor signaling pathways that are involved in lymphangiogenesis in OSCCa.

Copyright $\odot 2008$ S. Karger AG, Basel

\section{Introduction}

Lymphangiogenesis, the growth of lymphatic vessels, is considered to be an important process in the development of tumor metastases [1]. An increase in the number of lymphatic vessels in the tumor stroma has been shown to correlate with lymph node metastasis [2-4]. Several growth factors including vascular endothelial growth factor (VEGF), platelet-derived growth factor (PDGF), basic fibroblast growth factor (bFGF), insulin-like growth factors (IGF-I/II), and hepatocyte growth factor (HGF) have been reported to exhibit lymphangiogenic activity [5-12]. Furthermore, increased levels of some of these growth factors have been correlated with tumor lymphangiogenesis and lymph node metastasis in several tumors $[5,10,11]$.

The correlation between VEGF and tumor lymphangiogenesis in oral squamous cell carcinoma (OSCCa) has previously been reported [10]. However, little is known about the relationship between the expression of other

\section{KARGER}

Fax +41613061234 E-Mail karger@karger.ch www.karger.com
(C) 2008 S. Karger AG, Basel

1011-7571/08/0176-0486\$24.50/0

Accessible online at:

www.karger.com/mpp
Mohammad Abdulhadi Ali

Department of Diagnostic Sciences

Faculty of Dentistry, Kuwait University

Jabriya (Kuwait)

Tel. +965 498 6769, Fax +965 532 6049, E-Mail mali@hsc.edu.kw 
lymphangiogenic growth factors and lymphangiogenesis in OSCCa. Therefore an immunohistochemical analysis on OSCCa tissue samples was conducted using a panel of lymphangiogenic growth factors (VEGF, PDGF, bFGF, IGF-I, IGF-II, and HGF) to determine their expression and correlate the levels of expression with lymphatic microvessel density (LMVD).

\section{Materials and Methods}

\section{Tissue Specimens}

The original hematoxylin and eosin (HE)-stained sections and formalin-fixed, paraffin-embedded specimen blocks of all OSCCa cases derived from patients who were diagnosed between January 2004 and December 2006 were retrieved from the Department of Histopathology of Al-Amiri Hospital, Kuwait. The HE sections were reviewed to confirm the diagnosis. Cases with inadequate tissue, cases that lacked an epithelial/connective tissue interface, recurrent cases, and previously treated cases were excluded. A total of 25 OSCCa cases were selected for this study. Clinical information including age, gender, and site of involvement, were obtained from biopsy forms submitted by the clinicians. Of the 25 cases selected, 10 were from males and 15 were from females. Their mean age was 53 years ranging between 30 and 80 years. Nine cases were from the tongue, 8 from the buccal mucosa, 4 from the gingiva, 3 from the palate, and 1 from the floor of the mouth. The grade of OSCCa was determined according to the criteria of the WHO histological typing of oral and oropharyngeal tumors [13] and ranged from well-differentiated (grade I $=16$ cases) to moderately differentiated (grade II $=9$ cases) squamous cell carcinoma.

Blocks of 20 previously resected oral lesions containing normal oral epithelium, such as traumatic ulcers, frictional keratoses, and chronic mucositis, were used as controls. The control samples were selected such that they closely matched the OSCCa cases with respect to age, gender distribution, and tumor location. Of the 20 control samples, 8 were from males and 12 from females. The mean age of the control patients was 51.3 years, ranging between 33 and 70 years. Eight cases were from the tongue, 8 from the buccal mucosa, and 4 from the gingiva. The research protocol was approved by the Research Ethics Committee of the Health Sciences Center, Kuwait University, Kuwait.

\section{Immunohistochemical Staining}

For immunohistochemical analysis, tissue sections of $5 \mu \mathrm{m}$ thickness were prepared on coated slides (OptiPlus positivecharged slides BioGenex, San Ramon, Calif., USA). Sections were deparaffinized with xylene, rehydrated in graded alcohol, and washed in deionized water and phosphate-buffered saline. Heatinduced antigen retrieval was performed in a water bath. The slides were placed in a preheated Coplin jar containing Antigen Retrieval Citra Plus solution (BioGenex), and incubated at $95^{\circ} \mathrm{C}$ for $20 \mathrm{~min}$. The slides were then allowed to cool at room temperature for $20 \mathrm{~min}$.

Mouse monoclonal anti-PDGF and anti-bFGF (BioGenex) antibodies were used to detect PDGF and bFGF. A mouse monoclonal D2-40 (1:20) antibody (Signet, USA) was used to detect the lymphatic vessels. The Super Sensitive Multilink-HRP Detection $\mathrm{Kit} / \mathrm{DAB}$ (BioGenex) was used with these antibodies, and the staining procedure was performed according to the manufacturer's instructions. Sections were treated with peroxidase block (3\% hydrogen peroxide) to block endogenous peroxidase activity. Nonspecific protein-protein interactions were blocked by treating the tissue sections with Power Block (buffered casein solution). The sections were incubated in the prediluted primary antibody at room temperature, followed by incubation with multilink prediluted secondary antibody solution (biotinylated antiimmunoglobulins). The sections were treated with HRP label (horseradish peroxidase-conjugated streptavidin), followed by DAB chromogen/substrate (3,3'-diaminobenzidine chromogen/ $\mathrm{H}_{2} \mathrm{O}_{2}$ /substrate buffer) solution. The sections were counterstained with Mayer's hematoxylin solution, dehydrated, and mounted with dibutyl phthalate xylene. Each step was followed by washing/rinsing with phosphate-buffered saline.

Goat polyclonal anti-human VEGF, IGF-I, IGF-II, HGF antibodies and the Cell and Tissue Staining/HRP-DAB Detection Kit (R\&D Systems Inc., McKinley Place NE, Minneapolis, Minn., USA) were used to detect IGF-I, IGF-II, and HGF. Sections were treated with peroxidase block ( $3 \%$ hydrogen peroxide), and then treated with serum blocking reagent $D$. Nonspecific protein-protein interactions were blocked by treating the tissue sections with avidin and biotin blocking reagents. The sections were then incubated in the diluted primary antibody (IGF-I and IGF-II: $5 \mu \mathrm{g} / \mathrm{ml}$, VEGF and HGF: $10 \mu \mathrm{g} / \mathrm{ml}$ ) at room temperature, followed by incubation with a prediluted secondary antibody solution (biotinylated anti-goat secondary antibodies). The slides were then incubated in HSS-HRP label (high sensitivity streptavidin-horseradish peroxidase-conjugated). The sections were treated with $\mathrm{DAB}$ chromogen/substrate (3,3'-diaminobenzidine chromogen $/ \mathrm{H}_{2} \mathrm{O}_{2} /$ substrate buffer) solution, counterstained with Mayer's hematoxylin solution, dehydrated, and mounted with dibutyl phthalate xylene. Each step was followed by washing/rinsing with PBS. Each batch of staining was accompanied with positive and negative control slides.

\section{Evaluation of Growth Factor Expression, Lymphatic}

Microvessel Density, and Stromal Inflammation

To evaluate the immunoreactivity of the growth factors, the slides were placed in a random order, then examined and scored independently by the author and a colleague, Dr. Bobby Joseph (acknowledgment). In three randomly selected high-power fields $(\times 200)$, the intensity of cytoplasmic immunoreactivity in the squamous cells was evaluated based on a scale of 0 to $+3(0=$ no staining, $1=$ weak staining, $2=$ moderate staining, and $3=$ strongest intensity). The percentages of weakly, moderately, and strongly stained cells were determined and a staining score was calculated as follows: score (maximum of 300$)=$ sum of $0 \times$ percentage of negative cells, $1 \times$ percentage of weakly stained cells, $2 \times$ percentage of moderately stained cells, and $3 \times$ percentage of strongly stained cells, as described previously by Gombos et al. [4].

For determination of the LMVD, each D2-40-stained section was first scanned under a light microscope with low power magnification $(\times 40$ and $\times 100)$ to identify the areas with the highest number of lymphatic vessels. The number of lymphatic vessels within three high-power $(\times 200)$ fields of these areas was counted and an average count was recorded, as described by Weidner et al. [14]. 

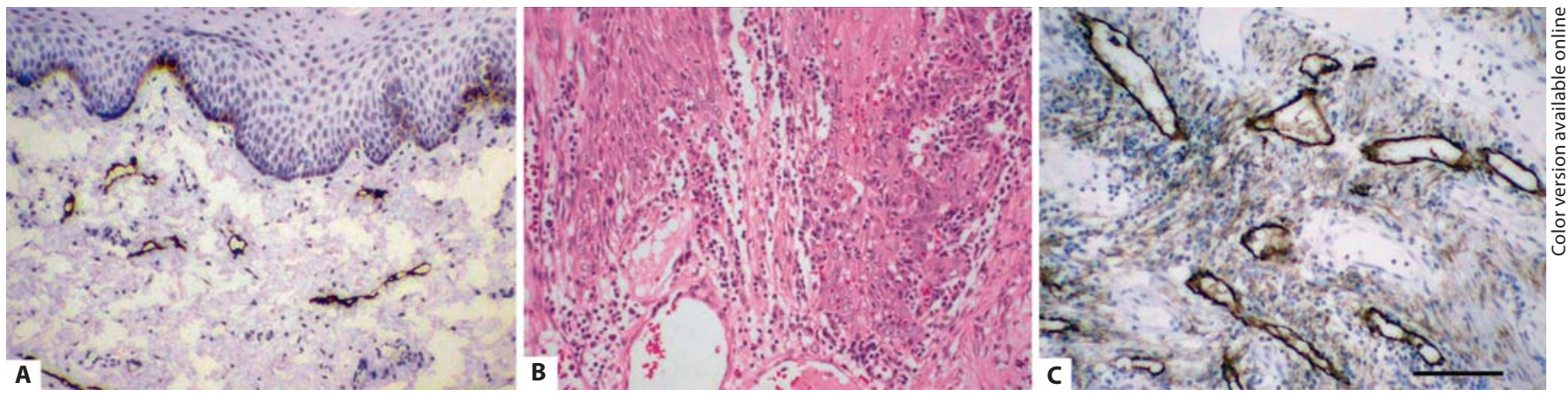

Fig. 1. LMVD staining pattern using D2-40 antibody. A Normal oral tissue with lymphatic microvessels located mainly in the superficial lamina propria. B HE-stained section of an OSCCa case showing the invasion front of the tumor. C LMVD staining pattern in the stroma of the OSCCa case shown in B. Scale bar $=100 \mu \mathrm{m}$.

HE-stained slides were examined to determine the degree of stromal inflammation in the adjacent connective tissue. Stromal inflammation was graded based on a scale of +1 to $+3(1=$ absent to sparse inflammatory infiltrate, $2=$ moderate/inhomogeneous infiltrate, and 3 = dense, homogeneous infiltrate), as described by Schoppmann et al. [15].

Statistical Analysis

Statistical analyses were performed using SPSS for Windows, version 12.0 (Chicago, Ill., USA). Interobserver reliability was assessed with Spearman's correlation coefficient. The Mann-Whitney test was used to compare the VEGF, PDGF, bFGF, HGF, IGFI, IGF-II, stromal inflammation, and LMVD levels between the OSCCa and normal tissues, and between the different histologic grades of OSCCa cases. For OSCCa samples, the Spearman's rank test was used to correlate growth factor expression and stromal inflammation with the LMVD. For all tests, a two-sided $p$ value of $<0.05$ was considered statistically significant.

\section{Results}

Lymphatic vessels were easily identified with D2-40 antibody. In normal oral mucosal tissue, the lymphatic vessels were relatively small and evenly distributed in the underlying superficial lamina propria. In OSCCa samples, the lymphatic vessels were more elongated and unevenly distributed in the surrounding stroma (fig. 1). D240 staining was also found in the basal cell layer of the epithelium. The LMVD in the stroma of OSCCa tissue was significantly higher than the LMVD associated with normal oral mucosa ( $\mathrm{p}<0.001$, Mann-Whitney test; table 1). No significant difference in LMVD existed between the different histologic grades of OSCCa cases $(\mathrm{p}=1.00$, Mann-Whitney test).

Eleven (55\%) normal oral mucosa cases showed grade 1 inflammation and $9(45 \%)$ cases showed grade 2 inflam- mation. Eight (32\%) OSCCa cases showed grade 1 inflammation, $15(60 \%)$ cases showed grade 2, and $2(8 \%)$ cases showed grade 3 inflammation. There was no significant difference in the amount of stromal inflammation between normal oral mucosa and OSCCa cases, or between the different histologic grades of OSCCa cases ( $p=0.08$ and $\mathrm{p}=0.559$, respectively, Mann-Whitney test).

Immunohistochemical expression of the growth factors was found in the cytoplasm of the epithelial cells. In normal oral mucosa, all cases showed some degree of positivity for VEGF, PDGF, bFGF, and IGF-II. Six cases were positive for HGF, and 4 cases were positive for IGFI, according to the criteria for immunohistochemical evaluation. In the OSCCa tissues, all 25 cases were positive for VEGF, PDGF, bFGF, HGF, and IGF-II and 23 cases were positive for IGF-I. As shown in table 1, the expression of VEGF, PDGF, bFGF, HGF, IGF-I, and IGF-II was significantly higher in the OSCCa tissues than in normal mucosa $(\mathrm{p}<0.001, \mathrm{p}<0.001, \mathrm{p}<0.001, \mathrm{p}<0.001, \mathrm{p}<$ 0.001 , and $\mathrm{p}<0.01$, respectively, Mann-Whitney test; fig. 2). No significant differences in the expression of VEGF, PDGF, bFGF, HGF, IGF-I, and IGF-II existed between the different histologic grades of OSCCa cases $(\mathrm{p}=0.487, \mathrm{p}=0.637, \mathrm{p}=0.487, \mathrm{p}=0.803, \mathrm{p}=0.169$, and $\mathrm{p}=0.718$, respectively, Mann-Whitney test). The interobserver correlation coefficient (between author and colleague) was $0.89(\mathrm{p}<0.001)$ for the LMVD data, 0.90 $(\mathrm{p}<0.001)$ for VEGF, $0.82(\mathrm{p}<0.001)$ for PDGF, 0.87 $(\mathrm{p}<0.001)$ for bFGF, 0.94 ( $\mathrm{p}<0.001)$ for HGF, 0.97 ( $\mathrm{p}<$ $0.001)$ for IGF-I, and 0.89 ( $\mathrm{p}<0.001)$ for IGF-II, indicating very good interobserver reliability.

In the OSCCa tissue, positive correlations (based on the Spearman correlation test) existed between LMVD and VEGF $(r=0.573 ; p<0.01)$ PDGF $(r=0.559 ; p<0.01)$, 
Fig. 2. Immunohistochemical staining of VEGF, PDGF, bFGF, HGF and IGF-I. A Histologically normal oral tissue showed strong VEGF staining in most of the epithelial cells. B An OSCCa showing strong VEGF staining in some of the tumor cells. C A normal oral tissue showing strong PDGF staining in some of the epithelial cells. D An OSCCa showing strong PDGF staining in most of the tumor cells. E A normal oral tissue showing no detectable immunoreactivity for bFGF. F An OSCCa showing strong bFGF staining in all tumor cells. G A normal oral tissue showing no detectable immunoreactivity for HGF. H An OSCCa showing moderate HGF staining in most of the tumor cells. I A normal oral tissue showing no detectable immunoreactivity for IGF-I. J An OSCCa showing moderate IGF-I staining in most of the tumor cells. Scale bar $=100 \mu \mathrm{m}$.
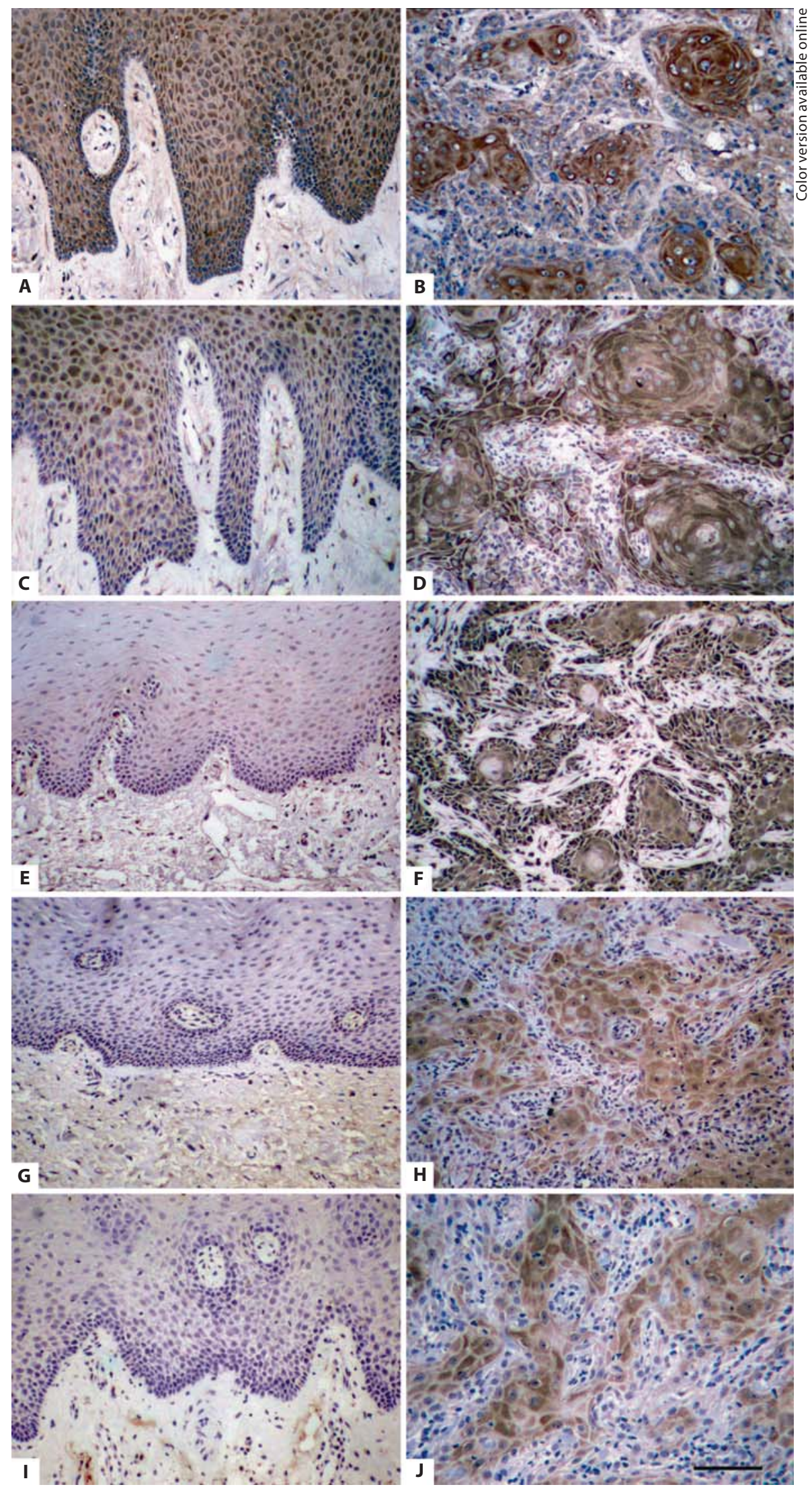
Table 1. Comparison between normal tissue and carcinoma by Mann-Whitney tests

\begin{tabular}{lccc}
\hline & Normal oral tissue $(\mathrm{n}=20)$ & OSCCa $(\mathrm{n}=25)$ & $\mathrm{p}$ value \\
\hline LMVD & $18.00(12.67-23.67)$ & $35.00(20.00-40.17)$ & 0.000 \\
VEGF expression & $94.17(33.33-133.33)$ & $162.50(123.67-210.00)$ & 0.000 \\
PDGF expression & $108.33(30.00-156.67)$ & $169.17(111.67-225.00)$ & 0.000 \\
FGF expression & $116.66(33.33-170.83)$ & $180.83(125.00-228.33)$ & 0.000 \\
HGF expression & $0.00(0.00-68.33)$ & $88.33(36.67-143.33)$ & 0.000 \\
IGF-I expression & $0.00(0.00-33.33)$ & $70.00(0.00-131.67)$ & 0.000 \\
IGF-II expression & $57.50(8.33-158.33)$ & $109.17(60.00-173.33)$ & 0.001 \\
\hline
\end{tabular}

Data shown as median with range in parentheses.

bFGF $(r=0.516 ; p<0.01)$, HGF $(r=0.448 ; p<0.05)$, and IGF-I $(r=0.6 ; p<0.01)$, but no correlations existed with IGF-II $(\mathrm{r}=0.380 ; \mathrm{p}=0.061)$, or with the stromal inflammation $(\mathrm{r}=0.161 ; \mathrm{p}=0.44)$.

\section{Discussion}

Previous studies have identified a number of growth factors that have lymphangiogenic activity [5-12]. Increased levels of some of these growth factors have been reported to correlate with tumor lymphangiogenesis and lymph node metastasis in several tumors $[5,10,11]$. VEGF is one of the most commonly studied lymphangiogenic growth factors. It has been shown to play a key role in tumor-associated lymphangiogenesis and lymphatic metastasis $[4,5,16,17]$. VEGF expression and the correlation between VEGF and tumor lymphangiogenesis in OSCCa have been reported [10]. The expression of other lymphangiogenic growth factors has been reported in OSCCa, but it was not correlated with tumor lymphangiogenesis $[18,19]$.

LMVD was used in this study as an indicator for lymphangiogenesis. The D2-40 antibody, a sensitive marker that distinguishes lymphatic vessels from vascular vessels [20], was used to measure LMVD in the adjacent stoma. In this study, the LMVD in OSCCa samples was significantly higher than in normal oral mucosa, which is consistent with the findings of Sedivy et al. [10] and Xie et al. [21]. No correlation existed between LMVD and tumor grade, a finding consistent with previous reports by Miyahara et al. [22] and Franchi et al. [2].

In addition, none of the growth factors studied were significantly correlated with tumor grade. There are differing opinions regarding the correlation between growth factor expression and OSCCa grade. Li et al. [23] reported that VEGF expression was correlated with tumor grade in OSCCa, while PDGF and bFGF expression were not correlated. Sedivy et al. [10] also reported a significant correlation between VEGF and tumor grade in OSCCa, while Smith et al. [24] showed no correlation between the same growth factor and tumor grade in oral and oropharyngeal squamous cell carcinoma. A lack of correlation between HGF and bFGF expression and the histologic grades of OSCCa has also been reported $[19,25]$.

In the current study, there was variation in the expression of lymphangiogenic growth factors in the epithelial cells of normal oral mucosa and OSCCa tissue. Generally, the expression was significantly higher in OSCCa tissue, which is consistent with previous reports $[18,19,23,26]$. Previous studies have shown that lymphangiogenic factors induce lymphangiogenesis through different signaling pathways [7-9, 27]. VEGF induces lymphangiogenesis through its VEGFR-3 receptor on lymphatic endothelial cells [16]. Its expression has been correlated with tumor metastasis via the lymphatic vessels in several human cancers including OSCCa $[4,26,28,29]$. HGF acts on the HGF-R (c-met) receptor, which is expressed on lymphatic endothelial cells [8]. It also has an indirect lymphangiogenic effect through the VEGFR-3 receptor [27]. HGF expression has previously been reported in several human cancers, and in some tumors its expression correlates with lymph node metastasis [12, 19, 30-33]. PDGF expression is also found in several human cancers, including OSCCa $[18,34,35]$. PDGF acts on PDGF receptors and the expression of PDGF by murine fibrosarcoma cells has been shown to correlate with tumor lymphangiogenesis and lymph node metastasis [7, 11]. b-FGF expression is found in head and neck tumors, including OSCCa [18], and reportedly induces lymphangiogenesis through the 
VEGFR-3 signaling pathway [6]. IGF-I/II expression is found in many human tumors and has been found to correlate with poor prognosis [36, 37]. IGF-II has been shown to correlate with lymph node metastasis in prostate cancer [38], whereas both IGF-I and IGF-II have been shown to induce lymphangiogenesis in mouse cornea [9]. It has been proposed that IGF-I and IGF-II may act as direct lymphangiogenic factors through their receptors and that their lymphangiogenic-inducing effects are independent of the VEGFR-3 signaling pathway [9].

Due to the association of tumor lymphangiogenesis with cancer metastasis and poor prognosis, inhibition of tumor lymphangiogenesis has become an important target for experimental anticancer drugs. Recent studies using antibodies or soluble fusion proteins that target some of these lymphangiogenic growth factors or their receptors have revealed that it is possible to block tumor-associated lymphangiogenesis $[5,6,16,27]$.

\section{Conclusion}

In this study, significant positive correlations were found between LMVD and VEGF, PDGF, FGF, HGF, and IGF-I in the OSCCa tissue, indicating that several growth factors may be responsible for the induction of lymphangiogenesis in OSCCa. These findings suggest that any future treatment for OSCCa utilizing antilymphangiogenic agents should target multiple growth factor signaling pathways that are involved in lymphangiogenesis.

\section{Acknowledgments}

This work was supported by Kuwait University through grant No. ZD02/06. Special thanks to Dr. Bobby Joseph (Department of Diagnostic Sciences, Faculty of Dentistry, Kuwait University) for the microscopic evaluation.

\section{References}

1 Stacker SA, Achen MG, Jussila L, Baldwin ME, Alitalo K: Lymphangiogenesis and cancer metastasis. Nat Rev Cancer 2002;2:573583.

-2 Franchi A, Gallo O, Massi D, Baroni G, Santucci M: Tumor lymphangiogenesis in head and neck squamous cell carcinoma: a morphometric study with clinical correlations. Cancer 2004;101:973-978.

- 3 Dadras SS, Lange-Asschenfeldt B, Velasco P, Nguyen L, Vora A, Muzikansky A, Jahnke K, Hauschild A, Hirakawa S, Mihm MC, Detmar M: Tumor lymphangiogenesis predicts melanoma metastasis to sentinel lymph nodes. Mod Pathol 2005; 18:1232-1242.

44 Gombos Z, Xu X, Chu CS, Zhang PJ, Acs G: Peritumoral lymphatic vessel density and vascular endothelial growth factor $\mathrm{C}$ expression in early-stage squamous cell carcinoma of the uterine cervix. Clin Cancer Res 2005; 11:8364-8371.

$\checkmark 5$ Stacker SA, Caesar C, Baldwin ME, Thornton GE, Williams RA, Prevo R, Jackson DG, Nishikawa S, Kubo H, Achen MG: VEGF-D promotes the metastatic spread of tumor cells via the lymphatics. Nat Med 2001;7: 186-191.

6 Kubo H, Cao R, Brakenhielm E, Makinen T, Cao Y, Alitalo K: Blockade of vascular endothelial growth factor receptor-3 signaling inhibits fibroblast growth factor-2-induced lymphangiogenesis in mouse cornea. Proc Natl Acad Sci USA 2002;99:8868-8873.
Vincent L, Rafii S: Vascular frontiers without borders: multifaceted roles of plateletderived growth factor (PDGF) in supporting postnatal angiogenesis and lymphangiogenesis. Cancer Cell 2004;6:307-309.

-8 Kajiya K, Hirakawa S, Ma B, Drinnenberg I, Detmar M: Hepatocyte growth factor promotes lymphatic vessel formation and function. EMBO J 2005;24:2885-2895.

-9 Björndahl M, Cao R, Nissen LJ, Clasper S, Johnson LA, Xue Y, Zhou Z, Jackson D, Hansen AJ, Cao Y: Insulin-like growth factors 1 and 2 induce lymphangiogenesis in vivo. Proc Natl Acad Sci USA 2005;102:1559315598.

10 Sedivy R, Beck-Mannagetta J, Haverkampf C, Battistutti W, Honigschnabl S: Expression of vascular endothelial growth factor- $\mathrm{C}$ correlates with the lymphatic microvessel density and the nodal status in oral squamous cell cancer. J Oral Pathol Med 2003;32:455460.

11 Björndahl MA, Religa P, Clasper S, Garvin S, Galter D, Meister B, Ikomi F, Tritsaris K, Dissing S, Ohhashi T, Jackson DG, Cao Y: PDGF-BB induces intratumoral lymphangiogenesis and promotes lymphatic metastasis. Cancer Cell 2004;6:333-345.

12 Kim CH, Moon SK, Bae JH, Lee JH, Han JH, Kim K, Choi EC: Expression of hepatocyte growth factor and c-Met in hypopharyngeal squamous cell carcinoma. Acta Otolaryngol 2006;126:88-94.

13 Wahi PN, Cohen B, Lutrha UK, Torloni $\mathrm{H}$ Histological Typing of Oral and Oropharyngeal Tumours, ed 4. Geneva, WHO, 1971, pp 17-188.
14 Weidner N, Semple JP, Welch WR, Folkman $\mathrm{J}$ : Tumor angiogenesis and metastasis: correlation in invasive breast carcinoma. $\mathrm{N}$ Engl J Med 1991;324:1-8.

-15 Schoppmann SF, Schindl M, BreitenederGeleff S, Soleima A, Breitenecker G, Karner B, Birner P: Inflammatory stromal reaction correlates with lymphatic microvessel density in early-stage cervical cancer. Anticancer Res 2001;21:3419-3423.

16 Karpanen T, Egeblad M, Karkkainen MJ, Kubo H, Ylä-Herttuala S, Jäättelä M, Alitalo $\mathrm{K}$ : Vascular endothelial growth factor $\mathrm{C}$ promotes tumor lymphangiogenesis and intralymphatic tumor growth. Cancer Res 2001; 61:1786-1790.

17 Hirakawa S, Kodama S, Kunstfeld R, Kajiya K, Brown LF, Detmar M: VEGF-A induces tumor and sentinel lymph node lymphangiogenesis and promotes lymphatic metastasis. J Exp Med 2005;201:1089-1099.

-18 Ninck S, Reisser C, Dyckhoff G, Helmke B, Bauer H, Herold-Mende C: Expression profiles of angiogenic growth factors in squamous cell carcinomas of the head and neck. Int J Cancer 2003;106:34-44.

19 Chen YS, Wang JT, Chang YF, Liu BY, Wang YP, Sun A, Chiang CP: Expression of hepatocyte growth factor and c-met protein is significantly associated with the progression of oral squamous cell carcinoma in Taiwan. J Oral Pathol Med 2004;33:209-217.

20 Kahn HJ, Bailey D, Marks A: Monoclonal antibody D2-40, a new marker of lymphatic endothelium, reacts with Kaposi's sarcoma and a subset of angiosarcomas. Mod Pathol 2002;15:434-440. 
-21 Xie ZJ, Yang XF, Fan J, Zhang YK, Wu QL, Gu ZY: The relationship between lymphangiogenesis and cervical lymph node micrometastasis in oral squamous cell carcinoma. Zhonghua Kou Qiang Yi Xue Za Zhi 2004;39: 221-223.

-22 Miyahara M, Tanuma J, Sugihara K, Semba I: Tumor lymphangiogenesis correlates with lymph node metastasis and clinicopathologic parameters in oral squamous cell carcinoma. Cancer 2007;110:1287-1294.

-23 Li C, Shintani S, Terakado N, Klosek SK, Ishikawa T, Nakashiro K, Hamakawa $\mathrm{H}$ : Microvessel density and expression of vascular endothelial growth factor, basic fibroblast growth factor, and platelet-derived endothelial growth factor in oral squamous cell carcinomas. Int J Oral Maxillofac Surg 2005;34: 559-565.

-24 Smith BD, Smith GL, Carter D, Sasaki CT, Haffty BG: Prognostic significance of vascular endothelial growth factor protein levels in oral and oropharyngeal squamous cell carcinoma. J Clin Oncol 2000;18:20462052.

-25 Forootan SS, Ke Y, Jones AS, Helliwell TR: Basic fibroblast growth factor and angiogenesis in squamous carcinoma of the tongue. Oral Oncol 2000;36:437-443.

-26 Wen Y, Yu D, Wang C, Li L, Wang X, Tang X, Fu F: Expression of VEGF-C and its correlation with cervical lymph nodes metastasis of oral cancers. Hua Xi Kou Qiang Yi Xue Za Zhi 2001;19:5-8.
27 Cao R, Björndahl MA, Gallego MI, Chen S, Religa P, Hansen AJ, Cao Y: Hepatocyte growth factor is a lymphangiogenic factor with an indirect mechanism of action. Blood 2006;107:3531-3536.

28 Nakamura Y, Yasuoka H, Tsujimoto M, Imabun S, Nakahara M, Nakao K, Nakamura M, Mori I, Kakudo K: Lymph vessel density correlates with nodal status, VEGF$\mathrm{C}$ expression, and prognosis in breast cancer. Breast Cancer Res Treat 2005;91:125132.

29 Kishimoto K, Sasaki A, Yoshihama Y, Mese H, Tsukamoto G, Matsumura T: Expression of vascular endothelial growth factor- $C$ predicts regional lymph node metastasis in early oral squamous cell carcinoma. Oral Oncol 2003;39:391-396.

-30 Takada N, Yano Y, Matsuda T, Otani S, Osugi H, Higashino M, Kinoshita H, Fukushima $\mathrm{S}$ : Expression of immunoreactive human hepatocyte growth factor in human esophagea squamous cell carcinomas. Cancer Lett 1995 97:145-148.

31 Suzuki K, Cheng J, Watanabe Y: Hepatocyte growth factor and c-Met (HGF/c-Met) in adenoid cystic carcinoma of the human salivary gland. J Oral Pathol Med 2003;32:8489.

32 Fukuura T, Miki C, Inoue T, Matsumoto K, Suzuki H: Serum hepatocyte growth factor as an index of disease status of patients with colorectal carcinoma. Br J Cancer 1998;78: 454-459.
33 Tanaka K, Miki C, Wakuda R, Kobayashi M, Tonouchi H, Kusunoki M: Circulating level of hepatocyte growth factor as a useful tumor marker in patients with early-stage gastric carcinoma. Scand J Gastroenterol 2004; 39:754-760.

34 Henriksen R, Funa K, Wilander E, Backstrom T, Ridderheim M, Oberg K: Expression and prognostic significance of plateletderived growth factor and its receptors in epithelial ovarian neoplasms. Cancer Res 1993;53:4550-4554.

35 Kawai T, Hiroi S, Torikata C: Expression in lung carcinomas of platelet-derived growth factor and its receptors. Lab Invest 1997;77: 431-436.

36 Werner H, LeRoith D: The role of the insulin-like growth factor system in human cancer. Adv Cancer Res 1996;68:183-223.

-37 Sohda M, Kato H, Miyazaki T, Nakajima M, Fukuchi M, Manda R, Fukai Y, Masuda N, Kuwano H: The role of insulin-like growth factor 1 and insulin-like growth factor binding protein 3 in human esophageal cancer. Anticancer Res 2004;24:3029-3034.

38 Mita K, Nakahara M, Usui T: Expression of the insulin-like growth factor system and cancer progression in hormone-treated prostate cancer patients. Int J Urol 2000;7: 321-329. 